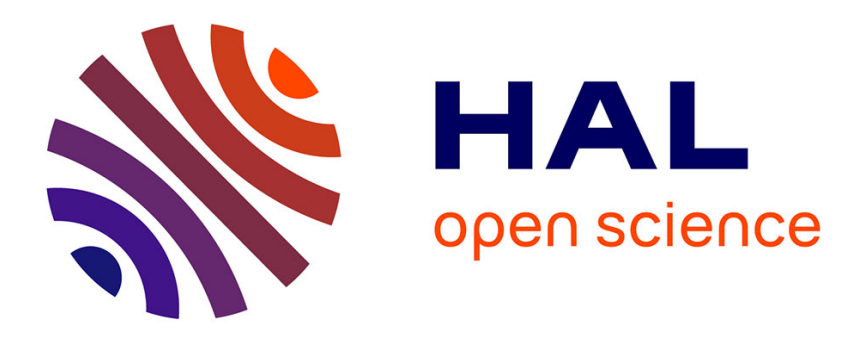

\title{
Un laser de grande puissance à milieu gazeux : le laser à iode
}

\author{
J.C. Guyot, J.C. Farcy, H. Guillet
}

\section{To cite this version:}

J.C. Guyot, J.C. Farcy, H. Guillet. Un laser de grande puissance à milieu gazeux: le laser à iode. Revue de Physique Appliquée, 1977, 12 (11), pp.1789-1796. 10.1051/rphysap:0197700120110178900 . jpa-00244403

\section{HAL Id: jpa-00244403 https://hal.science/jpa-00244403}

Submitted on 1 Jan 1977

HAL is a multi-disciplinary open access archive for the deposit and dissemination of scientific research documents, whether they are published or not. The documents may come from teaching and research institutions in France or abroad, or from public or private research centers.
L'archive ouverte pluridisciplinaire HAL, est destinée au dépôt et à la diffusion de documents scientifiques de niveau recherche, publiés ou non, émanant des établissements d'enseignement et de recherche français ou étrangers, des laboratoires publics ou privés. 


\title{
UN LASER DE GRANDE PUISSANCE A MILIEU GAZEUX : LE LASER A IODE $(*)(* *)$
}

\author{
J. C. GUYOT, J. C. FARCY \\ Division Lasers, Laboratoires de Marcoussis, \\ Centre de Recherches de la C. G. E., Route de Nozay, 91460 Marcoussis, France
}

\section{H. GUILLET}

Compagnie Industrielle des Lasers, route de Nozay, 91460 Marcoussis, France

(Reçu le 28 décembre 1976, révisé le 11 juillet 1977, accepté le 12 juillet 1977)

Résumé. - Le laser à iode, qui émet à 1,315 $\mu$, utilise habituellement la photolyse de composés iodés par rayonnement U. V. comme mécanisme de pompage. Par adjonction de gaz additifs $\left(\mathrm{Ar}, \mathrm{CO}_{2}\right)$, on peut ajuster sa section efficace d'émission stimulée à des valeurs $\left(2\right.$ à $\left.5 \times 10^{-19} \mathrm{~cm}^{2}\right)$ qui permettent de stocker des densités d'énergie d'environ $5 \mathrm{~J} / \mathrm{cm}^{2}$. Compte tenu des temps de relaxation entre les différents niveaux d'énergie, les rendements d'extraction d'énergie peuvent varier entre $67 \%$ et moins de $33 \%$. La possibilité d'obtenir des impulsions courtes $(<200 \mathrm{ps})$, de supprimer les énergies parasites et de produire des faisceaux de bonne qualité optique font du laser à iode un instrument certainement bien adapté aux expériences d'interaction lumière-matière. La puissance de $1 \mathrm{TW}$ (500 J en $500 \mathrm{ps)} \mathrm{récemment} \mathrm{obtenue} \mathrm{le} \mathrm{range} \mathrm{parmi} \mathrm{les} \mathrm{lasers} \mathrm{de} \mathrm{grande}$ puissance tels le laser à verre dopé au néodyme et le laser à $\mathrm{CO}_{2}$.

Abstract. - The iodine laser $(\lambda=1.315 \mu)$ uses flash photolysis of alkali-iodides as usual means of creating a population inversion. Through addition of foreign gas $\left(\mathrm{Ar}, \mathrm{CO}_{2}\right)$, the stimulated emission cross section can be adjusted to values $\left(2\right.$ to $\left.5 \times 10^{-19} \mathrm{~cm}^{2}\right)$ that allows to store energy densities of about $5 \mathrm{~J} / \mathrm{cm}^{2}$. Taking into account intra-levels relaxation time constants, the energy extraction efficiency is between $67 \%$ and less than $33 \%$. The ability to produce short pulses $(<200 \mathrm{ps})$, to suppress parasitic energies and to generate good optical quality beams allows the iodine laser to be a suitable tool for light-matter interaction experiments. A power output of $1 \mathrm{TW}(500 \mathrm{~J}-500 \mathrm{ps})$ has recently been obtained which puts this laser on the same level as other high-power lasers such as neodymium-doped glass and $\mathrm{CO}_{2}$ lasers.

Introduction. - Le laser à iode, découvert en 1964 par Kasper et Pimentel [1], n'a fait l'objet d'études et de développements systématiques qu'à partir de 1970, principalement au Max Planck Institut Für Plasmaphysik à Garching [2,3].

Les caractéristiques de ce laser sont telles qu'il est tout à fait envisageable de l'utiliser pour les expériences d'interaction lumière-matière qui réclament de grandes énergies, fournies en des temps courts. En effet, sa longueur d'onde d'émission $(\lambda=1,315 \mu)$ est proche du visible et d'ailleurs voisine de celle du laser à verre

$\left({ }^{*}\right)$ Cet exposé traite, en particulier, de travaux effectués avec l'appui du C. E. A., Division des Applications Militaires et de la Direction des Recherches et Moyens d'Essais.

$\left({ }^{* *}\right)$ Communication présentée au Congrès National de Physique des Plasmas, 6-10 décembre 1976. dopé au néodyme $(\lambda=1,06 \mu)$; sa section efficace d'émission stimulée permet de stocker l'énergie de façon aisée et il possède la faculté de restituer cette énergie en des temps courts, inférieurs à la nanoseconde.

Nous examinerons, dans ce qui suit, les principales caractéristiques physiques de ce laser, en portant l'accent sur celles qui intéressent directement son comportement en tant que laser de grande puissance.

Nous utiliserons les nombreux résultats obtenus dans les laboratoires étrangers, principalement ceux de Garching, ainsi que ceux obtenus à Marcoussis où les travaux portant sur le laser à iode ont débuté en fin d'année 1975.

1. Principaux mécanismes physiques du laser à iode. - 1.1 Les tRANSITIONS DU LASER A IODE. - La 
figure $1 a$ schématise les niveaux d'énergie donnant lieu à l'émission laser. La transition entre les niveaux ${ }^{2} \mathrm{P}_{1 / 2}$ et ${ }^{2} \mathrm{P}_{3 / 2}$ (nombre quantique principal $n=5$ ) est de $7603,15 \mathrm{~cm}^{-1}$, correspondant à une longueur d'onde de $1,315 \mu$ (soit un photon d'une énergie de $0,95 \mathrm{eV}$ ).
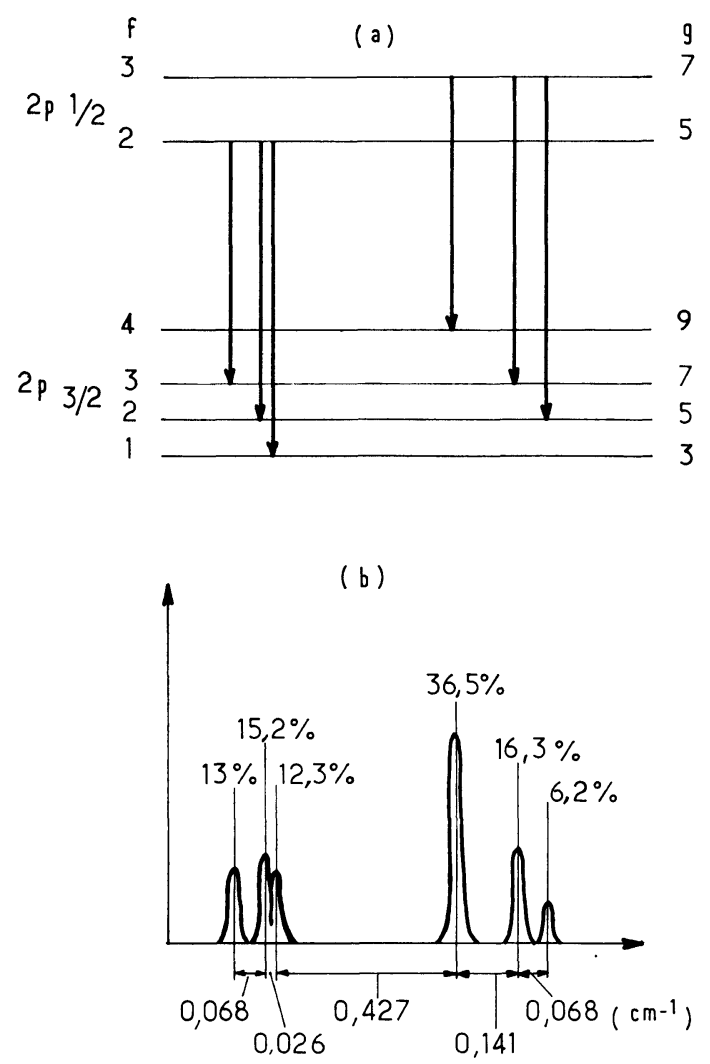

FIG. 1. - a) Niveaux d'énergie et transitions permises du laser à iode ; $b$ ) Intensité relative des raies de fluorescence. [a) Energy levels and allowed transitions of the iodine laser ; b) Relative intensity of fluorescence lines.]

Chacun de ces niveaux, correspondant aux deux valeurs possibles du moment angulaire électronique total $(J=L \pm S$, soit $3 / 2$ et $1 / 2)$ est lui-même décomposé en sous-niveaux.

En effet, le noyau de l'isotope 127 de l'iode possède un spin angulaire $I=5 / 2$. Son interaction avec le moment angulaire électronique total $J$ induit une structure hyper-fine, correspondant aux nombres quantiques $F$, tels que $\mathbf{F}=\mathbf{I}+\mathbf{J}(F=I \pm J)$. Il en résulte deux sous-niveaux de l'état ${ }^{2} \mathrm{P}_{1 / 2}(F=3,2)$ et quatre sousniveaux de l'état ${ }^{2} \mathrm{P}_{3 / 2}(F=4,3,2,1)$.

Ces sous-niveaux sont affectés de leurs poids statistiques respectifs, qui reflètent l'existence d'une sousstructure correspondant à une dégénérescence en $(2 I+1)(2 J+1)$ niveaux (soit un total de 12 niveaux hauts et 24 niveaux bas).

Les règles de sélection $(\Delta F=0, \pm 1)$ indiquent que le spectre d'émission comporte six raies (dont les plus extrêmes ne sont séparées que par $0,73 \mathrm{~cm}^{-1}$ ou $1,26 \AA$ ), la transition $F=3$ à $F=4$ étant la plus intense (Fig. 1b).
Les nombres quantiques associés aux différentes transitions permettent de calculer les probabilités d'émission spontanée de chacune des raies (voir tableau I) et conduisent à la probabilité d'émission totale :

$$
A=7,9 \mathrm{~s}^{-1} \text {. }
$$

\section{TABLEAU I}

Probabilité d'émission spontanée des six transitions permises

[Spontaneous emission rates for the six allowed transitions]

$$
\begin{aligned}
& \text { Transitions } \quad 3-4 \quad 3-3 \quad 3-2 \quad 2-3 \quad 2-2 \quad 2-1 \\
& \text { Probabilité } \\
& \begin{array}{lllllll}
\text { d'émission } \quad 5,08 & 2,2 & 0,63 & 2,46 & 3,07 & 2,37
\end{array} \\
& A_{i-j}\left(\mathrm{~s}^{-1}\right) \\
& A=\frac{g_{3}}{g_{2}+g_{3}}\left(A_{34}+A_{33}+A_{32}\right)+ \\
& +\frac{g_{2}}{g_{2}+g_{3}}\left(A_{23}+A_{22}+A_{21}\right)
\end{aligned}
$$

La durée de vie radiative correspondante est de $0,126 \mathrm{~s}$.

Cette valeur élevée est évidemment très favorable pour le stockage d'énergie.

1.2 DéComposition PhotolytiQue. - Traditionnellement, le laser à iode opère par dissociation photolytique de composés iodés [12], dont le plus communément utilisé est $\mathrm{C}_{3} \mathrm{~F}_{7} \mathrm{I}$.

Sous l'action du rayonnement U. V., la réaction suivante se produit:

$$
\mathrm{C}_{3} \mathrm{~F}_{7} \mathrm{I}+\mathrm{h} v(\mathrm{U} . \mathrm{V} .) \rightarrow \mathrm{C}_{3} \mathrm{~F}_{7}+\mathrm{I}\left({ }^{2} \mathrm{P}_{1 / 2}\right)
$$

suivie par l'émission laser, selon :

$$
\mathrm{I}\left({ }^{2} \mathrm{P}_{1 / 2}\right) \rightarrow \mathrm{I}\left({ }^{2} \mathrm{P}_{3 / 2}\right)+\mathrm{h} v(1,315 \mu) .
$$

La figure 2 montre le spectre d'absorption des principaux composés utilisés. Elle permet de constater que dans les conditions normales de température et de pression, le libre parcours moyen d'un photon U. V. sera inférieur à $1 \mathrm{~mm}$. Cette remarque permet de conclure que les pressions partielles du composé iodé devront être choisies en fonction du diamètre de l'amplificateur et que, dans les amplificateurs de grande dimension, il sera difficile d'obtenir à la fois une valeur élevée d'inversion de population, ainsi qu'une bonne uniformité radiale.

La méthode de production du rayonnement $\mathrm{U}$. V. nécessaire à la réaction (1) utilise le plus souvent des lampes à $\operatorname{arc}($ flash). Ces lampes, remplies de Xe à une pression de quelques dizaines de torr, sont traversées par des courants crêtes assez élevés (quelques kA), 


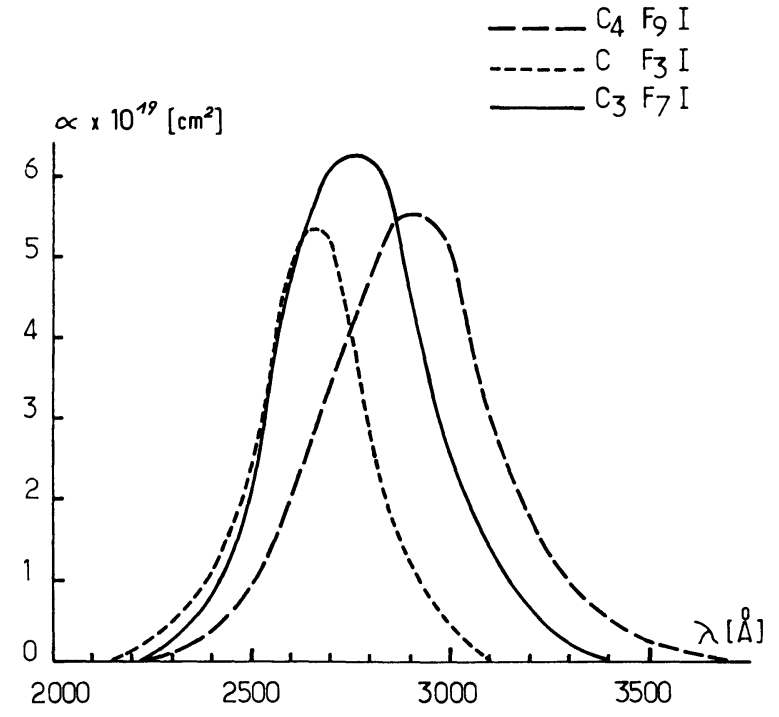

FIG. 2. - Spectre d'absorption des principaux composés de l'iode utilisés.

[Absorption spectrum of various alkali-iodides.]

nécessaires pour augmenter leur émission dans la partie U. V. du spectre. Il n'en reste pas moins que le rendement d'émission (rapport de l'énergie émise dans la bande $2500-2900 \AA \AA$ à l'énergie électrique injectée dans le flash) est assez mauvais [4] et compris entre $2 \%$ et $4 \%$. Ceci explique, en partie, le rendement encore peu élevé du laser à iode pompé par flash.

Des techniques d'amélioration de l'émission dans l'ultraviolet ont été démontrées [5], qui consistent en l'appoint d'additifs métalliques ou alcalins au Xe. Ces techniques se heurtent, cependant, à des difficultés technologiques (ségrégation des additifs).

D'autres méthodes de production de rayonnements U. V. ont été proposées et appliquées au pompage du laser à iode. Citons :

- l'utilisation de la fluorescence émise par les exciplexes d'halogénures et de gaz rare, $\mathrm{XeBr}$ en particulier [6] ;

- l'utilisation de l'émission d'une décharge induite par un fil explosif [7] ;

- l'utilisation du rayonnement U. V. d'un plasma créé par un laser $\mathrm{CO}_{2}[8]$.

Finalement, il est intéressant de mentionner que l'effet laser à $1,315 \mu$ a été obtenu dans des mélanges de $\mathrm{CF}_{3} \mathrm{I}$ et $\mathrm{N}_{2}$ excités par décharge électrique [9], produisant une dissociation électronique du $\mathrm{CF}_{3} \mathrm{I}$.

1.3 Désactivation. - Les atomes d'iode excités (niveaux ${ }^{2} \mathrm{P}_{1 / 2}$ ) peuvent perdre leur énergie non seulement par émission spontanée, mais encore par désactivation due aux collisions avec des gaz étrangers, suivant la réaction :

$$
\mathrm{I}^{*}+\mathrm{M} \stackrel{k_{M}}{\rightarrow} \mathrm{I}+\mathrm{M}
$$

La constante de désactivation $k_{\mathrm{M}}$ est particulièrement élevée [11] pour $\mathrm{O}_{2}$ et $\mathrm{I}_{2}$ (voir tableau II). Pratiquement, ceci nécessite, d'une part, de réaliser un vide relativement soigné $\left(10^{-2}-10^{-3}\right.$ torr) préalablement au remplissage des structures contenant le milieu actif, et, d'autre part, d'éliminer l'iode moléculaire qui est formé à la suite de la réaction de photolyse.

\section{TABLEAU II}

\section{Constantes caractéristiques de différents gaz intervenant dans le mélange actif}

[Quenching and collisional coefficients for various gases that can be present in the active mediums]

\begin{tabular}{|c|c|c|}
\hline$M$ & $\begin{array}{l}k_{\mathrm{M}} / 10^{-16} \\
\mathrm{~cm}^{3}-\mathrm{s}^{-1}\end{array}$ & $\begin{array}{c}\beta_{\mathrm{M}} / 10^{15} \\
\mathrm{~cm}^{-2}-\text { torr }^{-1}\end{array}$ \\
\hline $\mathrm{O}_{2}$ & $8,6 \times 10^{4}$ & \\
\hline $\mathrm{I}_{2}$ & $5 \times 10^{4}$ & \\
\hline $\mathrm{Ar}$ & $\leqslant 2 \times 10^{-2}$ & 3,6 \\
\hline $\mathrm{N}_{2}$ & 2 & 5,1 \\
\hline $\mathrm{CO}_{2}$ & 4,6 & 7,3 \\
\hline $\mathrm{C}_{3} \mathrm{~F}_{7} \mathrm{I}$ & 8,0 & 15,5 \\
\hline
\end{tabular}

La figure 3 montre la décroissance rapide de l'énergie laser (issue de l'oscillateur décrit au paragraphe 4.1 fonctionnant en relaxé multimode) avec le nombre de tirs lorsqu'on opère sans renouvellement des gaz : cette décroissance est due à la disparition progressive des molécules de $\mathrm{C}_{3} \mathrm{~F}_{7} \mathrm{I}$ et à l'apparition de molécules de $\mathrm{I}_{2}$.

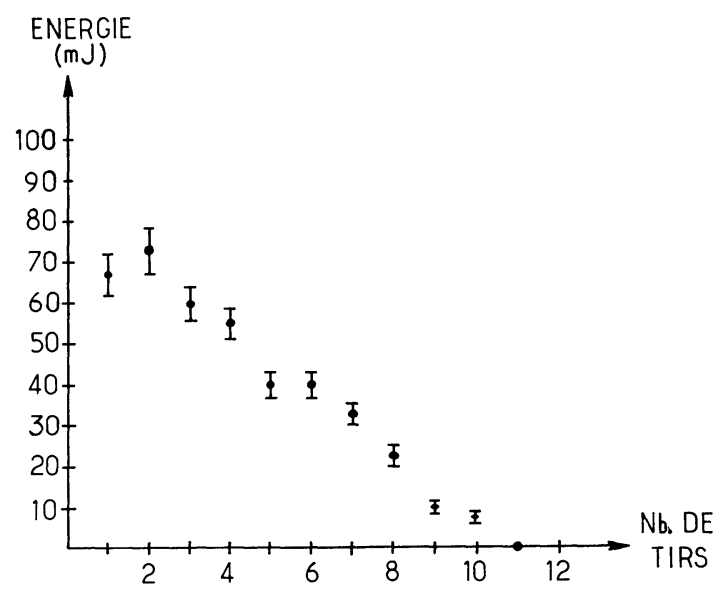

Fig. 3. - Evolution de l'énergie laser en fonction du nombre de tirs, sans renouvellement des gaz ( 25 torr de $\mathrm{C}_{3} \mathrm{~F}_{7} \mathrm{I}$ et $0,4 \mathrm{~kJ}$ d'énergie de pompage).

[Variation of output laser energy vs. number of shots, without gas recycling.]

Une méthode permettant d'opérer avec le laser à iode en circuit fermé a été développée [10], dont le principe est illustré sur la figure 4. Une pompe assure la cir- 


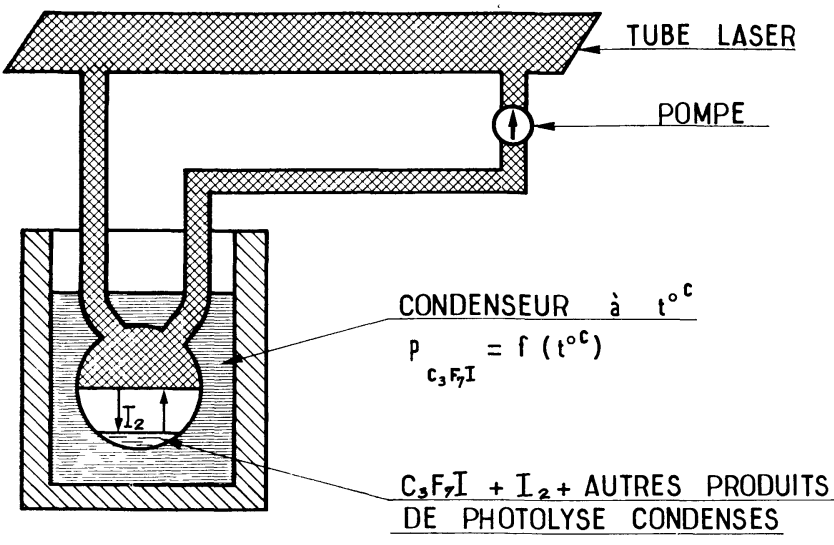

FIG. 4. - Principe du laser à iode fonctionnant en cycle fermé. [Schematic of a closed-loop iodine laser.]

culation des gaz à travers le système; la température du condenseur est réglée de façon à fournir la tension de vapeur de $\mathrm{C}_{3} \mathrm{~F}_{7} \mathrm{I}$ appropriée (pratiquement entre $-35^{\circ} \mathrm{C}$ et $\left.-5^{\circ} \mathrm{C}\right)$. A cette température, l'iode moléculaire se condense et est ainsi éliminé.

2. Stockage d'énergie. - Compte tenu des limitations imposées par le seuil de tenue des matériaux (aux environs de $5 \mathrm{GW} / \mathrm{cm}^{2}$ pour une impulsion de $1 \mathrm{~ns}$ ), il paraît inutile de stocker, dans le milieu actif, des énergies très supérieures à $5 \mathrm{~J} / \mathrm{cm}^{2}$. Les conditions opératoires usuelles permettent d'atteindre facilement cette valeur, compte tenu de la limitation imposée par les oscillations parasites.

2.1 LimitATION DUE AUX OSCILLATIONS PARASITES. Imaginons un amplificateur de longueur $L$ et de section droite $S$ (et donc de volume $V=S . L$ ).

Le gain à faible signal $G_{0}$ de cet amplificateur est donné par :

$$
G_{0}=\exp [\sigma \cdot \Delta N \cdot L]
$$

$\sigma=$ section efficace d'émission stimulée $\left(\mathrm{cm}^{2}\right)$;

$\Delta N=$ inversion de population (atomes excités $/ \mathrm{cm}^{3}$ ).

L'énergie stockée dans cet amplificateur $E_{\mathrm{s}}$ est donnée par :

$$
E_{\mathrm{s}}=h v \cdot \Delta N \cdot S \cdot L=\frac{h v}{\sigma} S \log \left[G_{0}\right]
$$

A partir de l'amplification de l'émission stimulée, un phénomène d'auto-oscillation peut apparaître, provoqué par des réflexions parasites (parois, poussières, etc...). Par exemple, des rayons issus du volume actif reviendront à leur point de départ après avoir subi des réflexions sur les fenêtres obturant l'amplificateur.

Dans ces conditions, le gain de l'amplificateur ne devra pas dépasser une valeur $G_{\max }$ pour éviter que la condition d'oscillation soit satisfaite, à savoir :

$$
G_{\max }^{2} R_{1} R_{2} T^{2}=1
$$

où $R_{1}$ et $R_{2}$ représentent des coefficients de réflexion et $T$ un coefficient d'atténuation.

Si la condition (5) est réalisée, l'énergie stockée dans l'amplificateur sera dissipée avant que l'impulsion issue de l'oscillateur ne vienne extraire cette énergie.

Il ressort, de la relation (4), que l'énergie maximum qui peut être stockée dans l'amplificateur est :

$$
E_{\mathrm{s}}^{\max }=\frac{h v S}{\sigma} \log \left[G_{\max }\right] .
$$

Dans la pratique, on constate qu'il est difficile de dépasser une valeur de $G_{\max }=10^{4}(40 \mathrm{~dB})$. Dans ces conditions, la relation (6) permet d'obtenir une valeur maximum de la densité d'énergie stockée par unité de surface d'amplificateur :

$$
e_{\mathrm{s}}^{\max }=\frac{13,9 \times 10^{-19}}{\sigma}
$$

exprimé en $\mathrm{J} / \mathrm{cm}^{2}$.

La relation (7) montre que l'énergie stockée dans un amplificateur peut être contrôlée si l'on sait ajuster la section efficace. En particulier, une diminution de cette dernière conduira à une augmentation de l'énergie stockée.

\subsection{AJUSTEMENT DE LA SECTION EFFICACE D'ÉMISSION} STIMULÉE. - C'est une des propriétés remarquables du laser à iode que la possibilité de déterminer, dans une certaine mesure, la valeur de la section efficace d'émission stimulée par ajustement de la largeur spectrale d'émission.

Cette largeur peut être modifiée [2] en utilisant un champ magnétique qui affecte la structure hyperfine des niveaux d'énergie en levant la dégénérescence.

Une seconde méthode, d'une mise en œuvre plus facile, utilise l'élargissement collisionnel [11].

En effet, dans des conditions où l'élargissement dû à la pression domine sur l'élargissement dû à l'effet Doppler, le profil d'émission est de Lorentz. Au centre de la raie d'émission, la largeur spectrale $\Delta v$ et la section efficace $\sigma$ sont liées par la relation :

$$
\sigma=\frac{\lambda^{2} A}{4 \pi^{2}} \frac{1}{\Delta v}
$$

où $A$ est la probabilité d'émission spontanée et $\lambda$ la longueur d'onde d'émission.

La largeur spectrale dépend elle-même de la pression

$$
\Delta v=\alpha_{0}+\sum_{i} \alpha_{i} p_{i}
$$

où $\alpha_{0}$ représente la faible contribution due à l'effet Doppler $(250-300 \mathrm{MHz})$ et $p_{i}$ les pressions partielles des gaz additifs, ayant des coefficients d'élargissement $\alpha_{\mathbf{i}}$.

Il ressort, des éqs. (8) et (9) que

$$
\frac{1}{\sigma}=\beta_{0}+\sum_{i} \beta_{i} p_{i}
$$


avec

$$
\alpha_{i}=\frac{\lambda^{2} A}{4 \pi^{2}} \beta_{i} .
$$

Dans ces conditions, l'énergie stockée croîtra linéairement avec la pression et la densité d'énergie stockée maximum s'écrira :

$$
\begin{aligned}
e_{\mathrm{s}}^{\max }=\frac{E_{\mathrm{s}}^{\max }}{S}=1,52 \times 10^{-19} & \log \left[G_{\max }\right] \times \\
& \times\left(\beta_{0}+\sum_{i} \beta_{i} p_{i}\right) .
\end{aligned}
$$

La figure 5 représente les résultats expérimentaux de la variation de $\sigma$ en fonction de la pression de $\mathrm{C}_{3} \mathrm{~F}_{7} \mathrm{I}$ et de celle de différents gaz additifs [11].

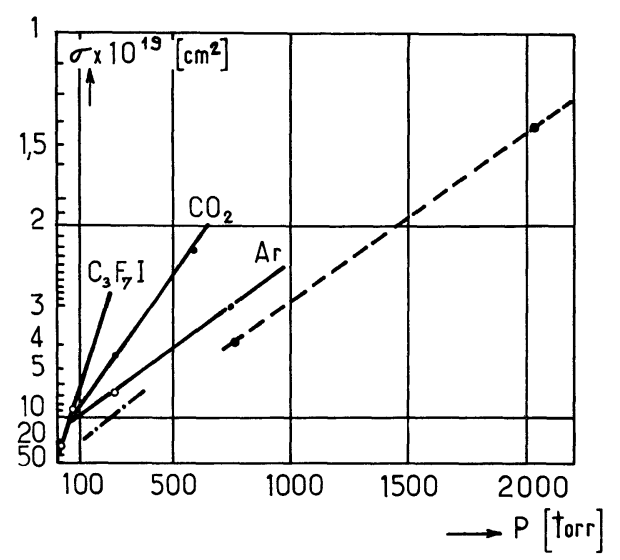

FIG. 5. - Variation de la section efficace d'émission stimulée en fonction de la pression. La courbe $\mathrm{C}_{3} \mathrm{~F}_{7} \mathrm{I}$ correspond au gaz pur, la courbe $\mathrm{CO}_{2}$ au mélange $\mathrm{CO}_{2}+50$ torr de $\mathrm{C}_{3} \mathrm{~F}_{7} \mathrm{I}$, la courbe $\mathrm{Ar}$ au mélange $\mathrm{Ar}+50$ torr de $\mathrm{C}_{3} \mathrm{~F}_{7} \mathrm{I}$. Les courbes en tireté et en pointillé correspondent à des mesures effectuées dans un mélange $\mathrm{Ar}+\mathrm{C}_{3} \mathrm{~F}_{7} \mathrm{I}$.

[Variation of the stimulated emission cross-section vs. pressure.]

A la suite des considérations évoquées au paragraphe 1.3, s'il est souhaitable d'utiliser des gaz d'appoint dont l'effet sur l'élargissement collisionnel soit important ( $\beta_{i}$ élevé), il faut que les constantes de désactivation soient faibles. A ce titre, $\mathrm{CO}_{2}, \mathrm{Ar}, \mathrm{N}_{2}$ (voir tableau II) représentent un bon compromis.

La figure 6 montre l'évolution de l'énergie stockée en fonction de la pression de gaz additif dans un amplificateur de gain $G_{\max }=10^{4}$, avec une pression partielle de $\mathrm{C}_{3} \mathrm{~F}_{7} \mathrm{I}$ prise égale à 10 torr.

Les courbes de la figure 6 sont tracées à partir de la relation (12) où il n'est pas tenu compte du chevauchement des raies qui apparaît aux pressions élevées ( $p \gg 1$ atm).

Des valeurs de l'énergie stockée égales à $5 \mathrm{~J} / \mathrm{cm}^{2}$ sont aisément accessibles avec des pressions de $\mathrm{CO}_{2}$ inférieures à la pression atmosphérique. Il n'est pas indiqué, d'ailleurs, de dépasser cette valeur au-delà de laquelle la désactivation due au $\mathrm{CO}_{2}$ deviendrait

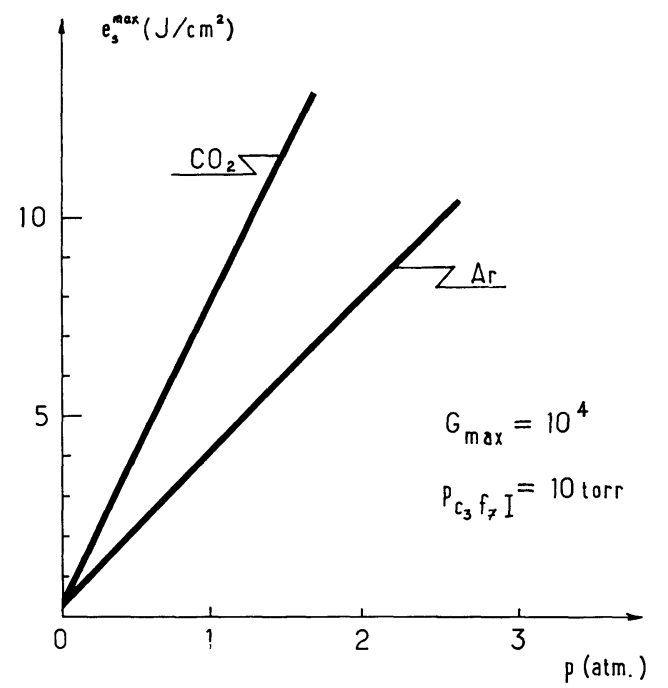

Fig. 6. - Variation de la densité d'énergie stockée en fonction de la pression totale dans un amplificateur de gain égal à $40 \mathrm{~dB}$, contenant 10 torr de $\mathrm{C}_{3} \mathrm{~F}_{7} \mathbf{I}$.

[Variation of the stored energy density vs. total pressure for an amplifier with $40 \mathrm{~dB}$ small-signal gain and 10 torr of $\mathrm{C}_{3} \mathrm{~F}_{7} \mathrm{I}$.]

trop importante ; il est alors préférable d'utiliser l'argon.

3. Extraction d'énergie. - Les rendements d'extraction d'énergie vont dépendre de la durée de l'impulsion laser vis-à-vis des temps de relaxation des niveaux de structure hyperfine.

En effet, les temps de relaxation $\tau_{2}$ entre les niveaux de l'état ${ }^{2} \mathrm{P}_{1 / 2}$ sont assez longs [12], allant de 1 à $10 \mathrm{~ns}$ dans les conditions opératoires habituellement utilisées. En outre, seuls les produits de photolyse (tels que $\mathrm{C}_{3} \mathrm{~F}_{7}$ et $I_{2}$ pour un laser opérant avec $C_{3} F_{7} I$ ) seront efficaces pour distribuer l'énergie entre ces niveaux, ce qui signifie qu'une augmentation de pression totale (grâce à un gaz additif) sera de peu d'effet.

Par contre, les temps de relaxation $\tau_{1}$ des niveaux de l'état ${ }^{2} \mathrm{P}_{3 / 2}$ sont relativement courts, compris entre 0,1 et $1 \mathrm{~ns}$.

Pour une inversion de population totale $\Delta N$ répartie sur les deux niveaux de l'état ${ }^{2} \mathrm{P}_{1 / 2}$, on peut, suivant différentes hypothèses, calculer le rendement d'extraction $\varepsilon$ connaissant l'inversion de population finale sur les niveaux en question. Ce rendement est défini par :

$$
\varepsilon=\frac{\text { Pop. initiale }- \text { Pop. finale }}{\text { Pop. initiale }}
$$

Le tableau III résume, schématiquement, les situations qui peuvent se présenter :

- Cas 1 : l'énergie est extraite sur l'ensemble des niveaux, dont les populations sont en équilibre, par une impulsion longue ( $\Delta T \geqslant 10 \mathrm{~ns})$. C'est aussi le cas où l'extraction se fait sur deux raies simultanément (au moyen d'un oscillateur travaillant à la fois sur les transitions 3-4 et 2-2) ou que l'impulsion a une très grande largeur spectrale. 


\section{TABLEAU III}

\section{Rendement d'extraction d'énergie et rendement théorique maximum suivant différentes valeurs de l'impulsion laser, comparée aux temps de relaxation}

[Energy extraction efficiency and maximum theoretical efficiency for different laser pulse durations compared to relaxation times]

\begin{tabular}{|c|c|c|c|c|}
\hline & $\begin{array}{l}\text { Population } \\
\text { initiale } \\
\end{array}$ & $\begin{array}{l}\text { Population } \\
\text { finale }\end{array}$ & $\begin{array}{l}\text { Rendement } \\
\text { d'extraction } \\
\qquad \underline{\varepsilon}\end{array}$ & $\begin{array}{c}\text { Rendement } \\
\text { théorique } \\
\text { maximum } \\
\rho=\eta \cdot \varepsilon \\
-\end{array}$ \\
\hline Cas $1: \Delta T>\tau_{1}, \tau_{2}$ & $\Delta N$ & $\frac{12}{36} \Delta N$ & $67 \%$ & $13,7 \%$ \\
\hline Cas 2: $\tau_{2}>\Delta T>\tau_{1}$ & $\frac{7}{12} \Delta N$ & $\frac{7}{12} \cdot \frac{7}{31} \Delta N$ & $45 \%$ & $9,2 \%$ \\
\hline Cas $3: \tau_{1}, \tau_{2}>\Delta T$ & $\frac{7}{12} \Delta N$ & $\frac{7}{12} \cdot \frac{7}{16} \Delta N$ & $\leqslant 33 \%$ & $\leqslant 6,7 \%$ \\
\hline \multicolumn{5}{|c|}{$\begin{aligned} \Delta N & =\text { inversion de population totale } \\
\tau_{2} & =\text { temps de relaxation des niveaux }{ }^{2} \mathrm{P}_{1 / 2}(1-10 \mathrm{~ns}) \\
\tau_{1} & =\text { temps de relaxation des niveaux }{ }^{2} \mathrm{P}_{3 / 2}(0,1-1 \mathrm{~ns})\end{aligned}$} \\
\hline
\end{tabular}

- Cas 2 : l'énergie est extraite sur un seul niveau (l'équilibre n'a pas été atteint entre les niveaux hauts) par une impulsion courte ( $\simeq 1 \mathrm{~ns}$ ), émise sur une seule transition (3-4).

- Cas 3 : l'équilibre n'est réalisé ni sur les niveaux hauts, ni sur les niveaux bas. L'énergie est extraite sur un seul niveau et le rendement dépendra de la cinétique des niveaux bas (embouteillage).

Sachant que le rendement quantique est donné par

$$
\eta=\frac{h v(2700)}{h v(13150)}=0,2
$$

on en déduit les rendements théoriques maxima $\rho=\eta \cdot \varepsilon$ dont les valeurs sont résumées dans le tableau III.

4. Production d'impulsions courtes. - Nous avons déjà souligné la possibilité qui existe d'ajuster, en jouant par exemple sur la pression totale du milieu actif, la largeur spectrale de la fluorescence $\Delta v$. Il est donc possible d'augmenter cette largeur, ce qui permet d'obtenir des impulsions courtes de durée $\Delta T$, la limite étant imposée par la relation d'incertitude

$$
\Delta v \cdot \Delta T=1 .
$$

4.1 Modulation active. - La technique la plus couramment utilisée est celle du blocage de modes au moyen d'une modulation active.

Cette méthode a été employée à Marcoussis, sur le montage expérimental schématisé sur la figure 7 , où l'on représente un oscillateur composé d'un tube de quartz de $8 \mathrm{~mm}$ de diamètre, contenant le milieu actif excité par 4 lampes à décharge montées en parallèle

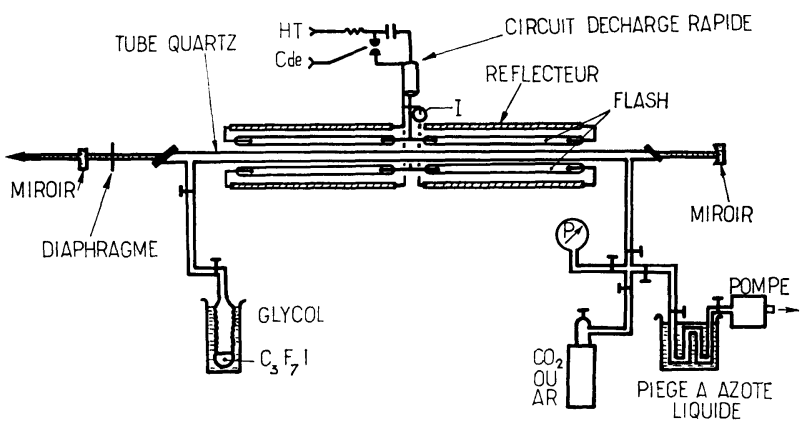

FIG. 7. - Schéma d'un oscillateur à iode.

[Schematic of a iodine laser oscillator.]

et entourées de réflecteurs. Dans le cas présent, cet oscillateur fonctionne sans recyclage des gaz : le $\mathrm{C}_{3} \mathrm{~F}_{7} \mathrm{I}$ est stocké à une température, régulée par une circulation de glycol, qui détermine la pression de remplissage. Le $\mathrm{C}_{3} \mathrm{~F}_{7} \mathrm{I}$ peut être additionné de gaz carbonique ou d'argon.

Ce tube est placé à l'intérieur d'une cavité optique de 3,75 m de long, dans laquelle on intercale un modulateur acousto-optique en silice, fonctionnant à $40 \mathrm{MHz}$, ce qui correspond à la fréquence propre de la cavité.

On obtient, dans ces conditions, des trains d'impulsions d'une durée de 100 à 150 ns, l'écart entre chaque impulsion étant d'environ $25 \mathrm{~ns}$. Pour une modulation sinusoïdale, telle que celle qui est utilisée, on peut montrer [13] que la durée de l'impulsion varie comme $(\Delta v)^{-1 / 2}$ et donc, en fonction de la pression totale (voir éq. (9)), comme $(\mathrm{p})^{-1 / 2}$.

La figure 8 indique la variation de la durée d'impulsion $\Delta T$ en fonction de la pression totale. Deux remarques s'imposent : 


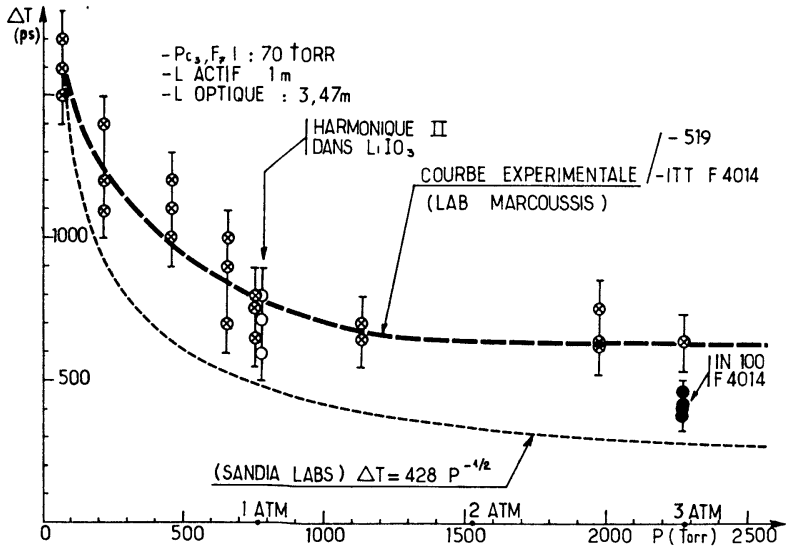

Fig. 8. - Variation de la durée d'impulsion, en fonction de la pression totale, dans un oscillateur à modulation acoustooptique.

[Variation of pulse duration vs. total pressure for an acoustooptical mode-locked oscillator.]

- la reproductibilité des impulsions n'est pas parfaite,

- le temps de montée de l'oscilloscope (300 ps) limite la résolution des mesures; à 1 atmosphère, la durée observée varie entre 600 et 800 ps, mais le temps de montée est celui de l'oscilloscope. La largeur de l'impulsion n'est donc pas parfaitement résolue.

A 3 atmosphères, des mesures opérées avec un oscilloscope rapide (IN 100) fournissent une durée d'impulsion de 400 ps.

Sur la même figure 8 , se trouvent représentés les résultats obtenus à Sandia Laboratories [14] où une durée minimum de 160 ps a été obtenue à une pression de 6500 torr.

Signalons, également, l'obtention d'impulsions de 300 ps grâce à une modulation sinusoïdale réalisée par un modulateur électro-optique [15].

4.2 Autres MÉThODES. - Il existe plusieurs autres méthodes permettant de produire des impulsions courtes. Citons :

a) la modulation passive : Cette méthode est liée à la découverte d'un absorbant saturable à $1,315 \mu$ dont le spectre d'absorption serait plus large que celui de la raie d'amplification et dont le temps de relaxation serait court ;

b) les obturateurs rapides : certains de ces obturateurs ont été expérimentés avec succès à d'autres longueurs d'onde, tels que :

- l'obturateur de Yablonovitch [16], utilisé pour le laser à $\mathrm{CO}_{2}$ et appliqué récemment au laser à iode pour produire des impulsions de 100 à 150 ps [17],

- l'obturateur de Duguay [18], dont la mise en œuvre est cependant difficile,

- l'obturateur à cellule électro-optique, utilisé couramment pour découper une impulsion courte à partir d'une impulsion longue de quelques dizaines de nanosecondes et qui est actuellement limité par les temps de montée des éclateurs qui lui sont associés ( $\simeq 300 \mathrm{ps}$ ).

Signalons qu'à $1,315 \mu$ la sensibilité des photocathodes $\mathrm{S} 1$ est très faible, ce qui rend la métrologie des impulsions rapides assez délicate quant à la mise en œuvre et à l'interprétation des résultats [19].

5. Contraste et énergies parasites. - Pour l'application aux expériences d'interaction matière-lumière, le rapport (contraste) entre le pic de puissance de l'impulsion principale et celui correspondant aux énergies parasites (pré-impulsions issues de l'oscillateur, émission stimulée amplifiée, etc...) doit être élevé $\left(\geqslant 10^{6}\right)$. Il est certain que la différence entre le gain à petit signal, suivant lequel sont amplifiées ces énergies parasites et le gain saturé, suivant lequel est amplifiée l'impulsion principale, tend à détruire le contraste au fur et à mesure de l'amplification.

Plusieurs méthodes sont utilisables pour créer un bon contraste ou l'améliorer. Citons :

a) Les cellules de Pockels : Un contraste de $10^{8} \mathrm{a} \mathrm{pu}$ être obtenu [20] en plaçant deux cellules de Pockels en série, à la sortie d'un oscillateur fonctionnant en blocage de modes.

b) Les absorbants saturables: A 1,315 $\mu$, un colorant (de composition inconnue) a été utilisé mais semble présenter un contraste assez faible [21].

L'iode atomique, dans son état fondamental, a également été utilisé en phase vapeur [22] (la transition ${ }^{2} \mathrm{P}_{3 / 2} \rightarrow{ }^{2} \mathrm{P}_{1 / 2}$ devient très absorbante à haute température). La saturation de l'absorption est facilement obtenue pour des densités d'énergie raisonnables (transmission égale à $90 \%$ pour $3-4 \mathrm{~J} / \mathrm{cm}^{2}$ ) tandis que l'absorption à bas niveau est bonne (environ $10^{3}$ pour une densité inférieure ou égale à $1 \mathrm{~mJ} / \mathrm{cm}^{2}$ ).

Une dernière source d'énergie parasite peut provenir de l'auto-oscillation de la chaîne amplificatrice. Pour éviter ce phénomène, il est nécessaire que les étages amplificateurs soient découplés les uns des autres : les absorbants saturables peuvent remplir cette fonction, ainsi que les rotateurs de Faraday qui assurent, en même temps, l'isolation de la chaîne contre les énergies réfléchies par les cibles.

6. Qualité du faisceau. - La qualité du faisceau issu d'un oscillateur, tel que celui décrit au paragraphe 4.1, est excellente. La figure 9 représente l'évolution du diamètre d'un faisceau gaussien en fonction de la distance et montre que cet oscillateur a une divergence proche de la valeur théorique. Ces mesures ont pu être effectuées grâce à une méthode d'hypersensibilisation des plaques photographiques KODAK I Z mise au point à Marcoussis [23].

Durant l'amplification, une dégradation de la qualité du faisceau peut apparaître : 


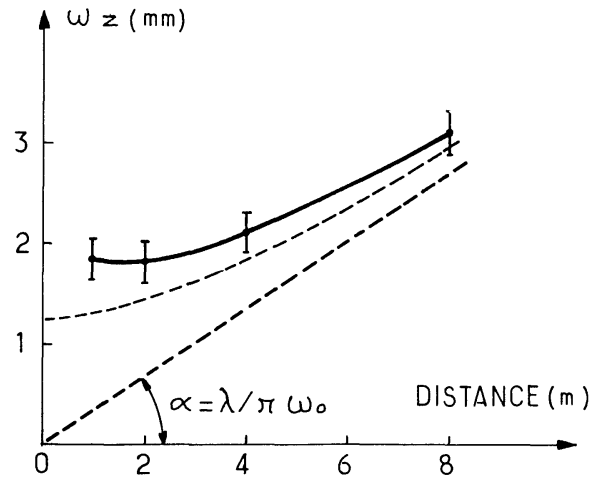

Fig. 9. - Evolution du diamètre du faisceau en fonction de la distance. En pointillé, la courbe théorique de croissance du diamètre d'un faisceau gaussien de diamètre minimum $\omega_{0}$. [Variation of beam diameter vs. distance. The dashed curve represents the theoretical variation of the diameter of a gaussian beam.]

- due aux phénomènes de diffraction, si le faisceau laser est intercepté par des diaphragmes, provoquant des anneaux de Fresnel. Ceci peut être efficacement combattu par l'apodisation [24] ;

- due à l'existence d'une onde de choc, issue des parois des amplificateurs et apparemment provoquée par la vaporisation des produits de photolyse sous l'action de la lumière des flash. Il ne faut, alors, utiliser qu'une partie de la section totale de l'amplificateur et synchroniser l'ensemble de la chaîne de façon que l'impulsion laser soit passée avant que l'onde de choc, se dirigeant vers le centre, n'atteigne les limites du volume actif utile;

- due aux phénomènes non linéaires. Le milieu actif étant gazeux, son indice non linéaire est très inférieur à celui d'un solide comme le verre, par exemple, où un tel indice a une influence profonde sur la qualité du faisceau [25]. Des essais préliminaires [26] ont montré qu'aucun effet anormal n'était observé jusqu'à des puissances de $10 \mathrm{GW} / \mathrm{cm}^{2}$.

Il ressort, cependant, que des mesures systématiques restent à faire pour évaluer les rendements de focalisa- tion (rapport de l'énergie focalisée dans un angle faible, cinq fois la limite de diffraction, par exemple, à l'énergie émise par le laser). Signalons, toutefois, que les premières expériences de focalisation sur cible [27] ont permis d'atteindre des densités de puissance d'environ $10^{15} \mathrm{~W} / \mathrm{cm}^{2}$, sur un diamètre de $40 \mu$ (correspondant à environ 4 fois la limite de diffraction).

7. Conclusion. - Le laser à iode a, d'ores et déjà, atteint des énergies et des puissances [28] (500 J en $500 \mathrm{ps}$, soit $1 \mathrm{TW}$ ) comparables à celles obtenues sur les lasers de grande puissance à gaz carbonique ou à verre dopé au néodyme.

Il possède, effectivement, les attributs que l'on peut souhaiter pour effectuer les expériences d'interaction lumière-matière, sous réserve de confirmer deux points importants : la bonne qualité du faisceau aux hautes énergies et la possibilité d'obtenir un bon contraste en sortie de la chaîne amplificatrice.

Les avantages qu'il semble posséder vis-à-vis du laser à verre dopé au néodyme devraient être très prochainement confirmés. Signalons déjà que, pour une puissance crête comparable, se situant au niveau de $1,5 \mathrm{TW}$, la chaîne laser à verre ARGUS de Livermore (U. S. A.) utilise une énergie électrique de pompage [29] quatre fois supérieure au laser ASTERIX III installé au Max Planck Institut (1,6 MJ contre 0,4 MJ).

Vis-à-vis du laser à gaz carbonique, dont le rendement reste encore supérieur, il possède, par contre, l'avantage d'émettre à une longueur d'onde peut-être plus intéressante en ce qui concerne le couplage de l'énergie laser à la cible, et relève d'une technologie sans doute moins onéreuse et plus fiable que celle qui nécessite des canons à électrons comme méthode d'injection de l'énergie pompage.

Enfin, le laser à iode est un laser à gaz : à ce titre, il est certainement capable de fonctionner avec une certaine cadence de répétition, ce qui fait de lui un candidat possible pour l'étape de faisabilité technique de production de puissance moyenne à partir de réactions thermonucléaires contrôlées.

\section{Bibliographie}

[1] Kasper, J. V., Pimentel C. G. Appl. Phys. Lett. 5 (1964) 231.

[2] HoHLa, K., Rapport IPP IV/33 (1971).

[3] Hohla, K. et coll,. J. Appl. Phys. 46 (1975) 808.

[4] Gusinow, M. A., IEEE J. Quant. Electron. 11 (1975) 929.

[5] Gusinow, M. A., Appl. Opt. 14 (1975) 2645.

[6] Swingle, J. C. et coll., Appl. Phys. Lett. 28 (1976) 387

[7] Basov, N. G. et Zuev, V. S., Nuovo Cimento 31 (1976) 129.

[8] Silfvast, W. T. et coll., Appl. Phys. Lett. 25 (1974) 593.

[9] Pleasance, L. D. et Weaver, L. A., Appl. Phys. Lett. 27 (1975) 407.

[10] Fuss, W. et Hohla, K., Opt. Commun. 18 (1976) 427.

[11] Fuss, W. et HoHLA, K., Z. Naturforsch. A. 31A (1976) 569.

[12] DAvis, C. C. et coll., IEEE J. Quant. Electron. 12 (1976) 334.

[13] Siegmann, A. E. et Kuizenga, O. J., Opt. Electron. 6 (1974) 43.

[14] Jones, E. D. et coll., Opt. Quant. Electron. 8 (1976) 231.

[15] Baker, H. J. et King, T. A., J. Phys. E : Scientific Inst. 9 (1976) 287.
[16] Yablonovitch, E., Phys. Rev. A 10 (1974) 1888.

[17] Hohla, K., Communication privée.

[18] Duguay, M. A., Appl. Phys. Lett. 15 (1969) 192.

[19] Baker, H. J. et KING, T. A., Opt. Commun. 18 (1976) 286.

[20] Hohla, K., Communication privée.

[21] Gal'Pern, M. G. et cull., Sou. J. Quant. Electron. 5 (1976) 1384.

[22] Fill, E. et Hohla, K., Opt. Commun. 18 (1976) 431.

[23] Farcy, J. C. et Beaupere, D., Rev. Sci. Instrum. 8 (1977) 11.

[24] Campillo, A. J. et coll., Opt. Commun. 10 (1974) 313.

[25] GuYot, J., Betringer, A., Auric, D., Congrès National de Physique des Plasmas, Paris (1976)

[26] Hohla, K., 9th Intern. Conf. on Quant. Elect. Amsterdam (1976).

[27] EidmanN, K. et coll., European Conference on Laser Interaction with matter Palaiseau (1976) J. Physique Colloq. 38 (1977) C 5.

[28] WitTE, K. et coll., 4th Int. Conf. on Laser Interaction and Related Phenomena Troy (1976).

[29] Glaze, J. A. et coll., Rapport UCRL, 77944 (1976). 